\title{
A Strict Control Lyapunov Function for a Diffusion Equation with Time-Varying Distributed Coefficients
}

\author{
Federico Bribiesca Argomedo, Christophe Prieur, Emmanuel Witrant and Sylvain Brémond
}

\begin{abstract}
In this paper, a strict Lyapunov function is developed in order to show the exponential stability and input-to-state stability (ISS) properties of a diffusion equation for nonhomogeneous media. Such media can involve rapidly time-varying distributed diffusivity coefficients. Based on this Lyapunov function, a control law is derived to preserve the ISS properties of the system and improve its performance. A robustness analysis with respect to disturbances and estimation errors in the distributed parameters is performed on the system, precisely showing the impact of the controller on the rate of convergence and ISS gains. This is important in light of a possible implementation of the control since, in most cases, diffusion coefficient estimates involve a high degree of uncertainty. An application to the safety factor profile control for the Tore Supra tokamak illustrates and motivates the theoretical results. A constrained control law (incorporating nonlinear shape constraints in the actuation profiles) is designed to behave as closely as possible to the unconstrained version, albeit with the equivalent of a variable gain. Finally, the proposed control laws are tested under simulation, first in the nominal case and then using a model of Tore Supra dynamics, where they show adequate performance and robustness with respect to disturbances.
\end{abstract}

\section{INTRODUCTION}

\section{Theoretical Contribution}

Parabolic partial differential equations (PDEs), and in particular diffusion or diffusion-convection equations, are used to model a wide array of physical phenomena ranging from heat conduction to the distribution of species in biological systems. While the diffusivity coefficients can be assumed to be constant throughout the spatial domain for most applications, spatially-distributed coefficients are needed when treating nonhomogeneous or anisotropic (direction-dependent) media. Unfortunately, extending existing results from the homogeneous to the nonhomogeneous case is not straightforward, particularly when the transport coefficients are timevarying.

In this article, the concept of input-to-state stability (ISS) will be the chosen framework to study the stability and robustness of a diffusion equation in a circular domain under a revolution symmetry condition with symmetric initial conditions. The interest of studying such an equation is illustrated and motivated by the proposed application, where a similar equation arises from the averaging of a 2-D physical equation

F. Bribiesca Argomedo, C. Prieur and E. Witrant are with Université de Grenoble / UJF / CNRS, GIPSA-lab UMR 5216, BP 46, F-38402 St. Martin D'Hères, France. Email: \{federico.bribiesca-argomedo, christophe.prieur, emmanuel.witrant\}egipsa-lab. grenoble-inp.fr

S. Brémond is with CEA, IRFM F-13108, Saint Paul-lez-Durance, France. Email: sylvain.bremond@cea.fr (representing the evolution of the toroidal magnetic flux in a tokamak) over the angle at fixed radius (nested toroidal surfaces). A comprehensive survey of ISS concepts, in the finite-dimensional case, can be found in [30]. ISS essentially implies guaranteeing a bounded gain between disturbances or errors and the state of the system. ISS-like properties in the infinite dimensional framework using a frequency-domain approach can be found for example in [15]. Nevertheless, we have favored the use of a Lyapunov-based approach to allow for an easier treatment of very general disturbances and errors in the system.

Although the use of Lyapunov functions in an infinite dimensional setting is not new, see for example [3], it is still an active research topic. Some interesting results for parabolic PDEs can be mentioned: [8], where a Lyapunov function is used to prove the existence of a global solution to the heat equation; [16], where a Lyapunov function is constructed for the heat equation with unknown destabilizing parameters (and subsequent control extensions [28] and [29]). Lyapunov based approaches are not limited to parabolic PDEs: Lyapunov functions are used in [10] for the stabilization of a rotating beam; in [9] for the stability analysis of quasilinear hyperbolic systems and in [11] for the construction of stabilizing boundary controls for a system of conservation laws. In particular, in [19] and [25] the interest of using a strict-Lyapunov function to obtain ISS-like properties is discussed in the parabolic and hyperbolic cases, respectively. The use of weighted $L^{2}$ norms (or similar quadratic expressions with a weight) as Lyapunov functions is not new and a few examples can be found in [23] (for time delay systems) and [12] (where a vanishing weight is also used for the control of the magnetic flux equation in a tokamak but not its gradient).

Some previous works on reaction-diffusion equations in cylindrical 2-D domains are, for instance, [32] and [34] in which boundary control laws are developed for the stabilization of thermal convection loops. However, in both of these articles the domain considered does not include the point $r=0$, which implies that none of the coefficients in the equation are singular.

In this article, we develop a strict Lyapunov function for the diffusion equation for a certain set of diffusivity coefficient profiles. Our main contribution is that the coefficients are allowed to be space and time dependent without imposing any constraints on the rate of variation of the coefficients with respect to time. This is an improvement over other works that consider diffusivity coefficients as being space-dependent or time-varying but not both simultaneously. Examples of such approaches are provided by [26], where constant dif- 
fusion coefficients and distributed convection coefficients are considered; [27], where the case of non-constant diffusion coefficients is treated (for continuous, time-invariant coefficients); or [33], where distributed and time-varying convection coefficients are taken into account (with a constant diffusion coefficient). Also, stability and robustness of the system under a simple unconstrained feedback law (that includes the open-loop system as a limiting case) were derived from the Lyapunov function, with results addressing most sources of errors and uncertainties that may be present in a real system. In particular, the following sources of error were considered:

- state disturbances: accounting for example for unmodeled dynamics;

- actuation errors: accounting mainly for errors in the actuator models (similar to the concept of controller fragility);

- estimation errors in the state and diffusivity coefficients: accounting for instance for discretized measurements or uncertain models, as well as measurement noise.

\section{Application to the Control of a Tokamak Safety Factor}

The motivating application for the theoretical results presented in this article is the development of a strict Lyapunov function and control laws for the poloidal magnetic flux profile in the Tore Supra tokamak. This application is particularly interesting for the method developed in this article since the diffusivity coefficient profiles depend mainly on the temperature profile inside the plasma, which is rapidly time-varying (more than ten times faster than the magnetic flux dynamics), and also on other physical quantities (like particle density and effective electric charge) that induce large model uncertainties and unmeasured disturbances. Furthermore, neglected inputs and unmodeled dynamics provide other sources of disturbances. The robustness results obtained from our theoretical contribution allow us to construct a constrained control law that will preserve ISS properties while taking into account strong nonlinear shape constraints in the distributed control action.

A tokamak is a toroidal chamber lined with magnetic coils that generate a very strong magnetic field with both a toroidal and a poloidal component. In this chamber, a plasma (generally constituted of Hydrogen isotopes) is confined by strong magnetic fields so that the fusion reaction can take place. The relation between the two components of the associated magnetic flux determines what is known as the safety factor profile or q-profile. This important physical quantity has been found to be related to several phenomena in the plasma, in particular magnetohydrodynamic (MHD) instabilities. Having an adequate safety factor profile is particularly important to achieve advanced tokamak operation, providing high confinement and MHD stability. A detailed account of tokamak physics can be found in [36]. An overview of challenges of tokamak plasma control is given in [24] and [35].

The problem of poloidal magnetic flux profile control is closely related, via the Maxwell equations, to the control of current profiles in the plasma. Some previous results in this areas can be found in [17], where experimentally identified linear models based on a Galerkin projection are used to control multiple profiles in JET; [20], where a reduced-order linear model is used to control some points in the safety factor profile; and in [22] among other papers, where an infinitedimensional model is used to construct an optimal feedback controller for the current profile, albeit considering a fixed form profile for the current deposit from the actuators and a good knowledge of the diffusivity profile.

In particular, some works related to Tore Supra are: [21], with an overview of control achievements; [6], where a polytopic LPV approach is used to build a common Lyapunov function guaranteeing stability of the discretized system with time-varying coefficients; [12], where sum-of-square polynomials are used to construct a Lyapunov function considering constant diffusivity coefficients and [13], where a sliding-mode controller is designed on the infinite-dimensional system, considering constant diffusivity coefficients.

For the application, the method proposed in this article has the advantage of not only considering the diffusivity coefficients as uncertain, but also of not bounding their rate of time-variation, thus reflecting the actual plasma physics in which the temperature evolves in a much faster timescale than that of the flux diffusion. A deep robustness analysis has been carried out with respect to different sources of error that have a prime importance in the physical system. Finally, nonlinear constraints in the actuators (representing the complex coupling between the plasma and the input wave generated by the radiofrequency antenna) that do not assume a constant Lower Hybrid current source deposit profile (contrarily to previous works) are introduced.

This paper is organized as follows. In Section II, the reference diffusion equation is presented and the existence and uniqueness of sufficiently regular solutions with timevarying coefficients is stated. Next, in Section III the main result is presented, namely the strict Lyapunov function and sufficient conditions for exponential stability of the system in an $L^{2}$ sense. In Section IV results are obtained regarding the robustness of the system with respect to several sources of errors and disturbances. In Section V the results are applied to the control of the poloidal magnetic flux profile in the Tore Supra tokamak and actuator constraints are added in such a way as to preserve ISS properties and to maximize the convergence rate of the system within some admissible limits.

\section{Problem Statement and Existence Results}

The diffusion equation considered in this article, in its polar representation with a revolution symmetry (angle independence) constraint is ${ }^{1}$ :

$$
\begin{array}{r}
\zeta_{t}(r, t)=\frac{\eta(r, t)}{r}\left[r \zeta_{r}(r, t)\right]_{r}+\eta(r, t) u(r, t), \\
\forall(r, t) \in(0,1) \times[0, T)
\end{array}
$$

\footnotetext{
${ }^{1}$ In this article, for any function $\xi$ depending on $r$ and/or $t, \xi_{r}$ and $\xi_{r r}$ are used to denote $\frac{\partial}{\partial r} \xi$ and $\frac{\partial^{2}}{\partial r^{2}} \xi$, respectively; $\dot{\xi}$ represents $\frac{d}{d t} \xi$ and $\xi^{\prime}$ represents $\frac{d}{d r} \xi$.
} 
with Neumann boundary conditions:

$$
\begin{aligned}
& \zeta_{r}(0, t)=0, \forall t \in[0, T) \\
& \zeta_{r}(1, t)=0, \forall t \in[0, T)
\end{aligned}
$$

and initial condition:

$$
\zeta(r, 0)=\zeta_{0}(r), \forall r \in(0,1)
$$

where $\eta$ stands for the diffusivity coefficient, $\zeta(\cdot, t)$ is the state of the system at time $t, u(\cdot, t)$ is a distributed input which can be either a control, a disturbance, or the sum of both, $0<T \leq+\infty$ is the time horizon. Hereafter, the dependence of $\zeta, u$ and $\eta$ on $(r, t)$ will be implicit and omitted in most equations.

The following properties are assumed to hold in (1):

$\mathbf{P}_{\mathbf{1}}: \eta(r, t) \geq k>0$ for all $(r, t) \in[0,1] \times[0, T)$.

$\mathbf{P}_{2}$ : The two-dimensional Cartesian representations of $\eta$ and $u$ are $\operatorname{in}^{2} C^{1+\alpha_{c}, \alpha_{c} / 2}(\bar{\Omega} \times[0, T]), 0<\alpha_{c}<1$, where $\Omega \doteq\left\{\left(x_{1}, x_{2}\right) \in \mathbb{R}^{2} \mid x_{1}^{2}+x_{2}^{2}<1\right\}$ as shown in Fig. 1 .

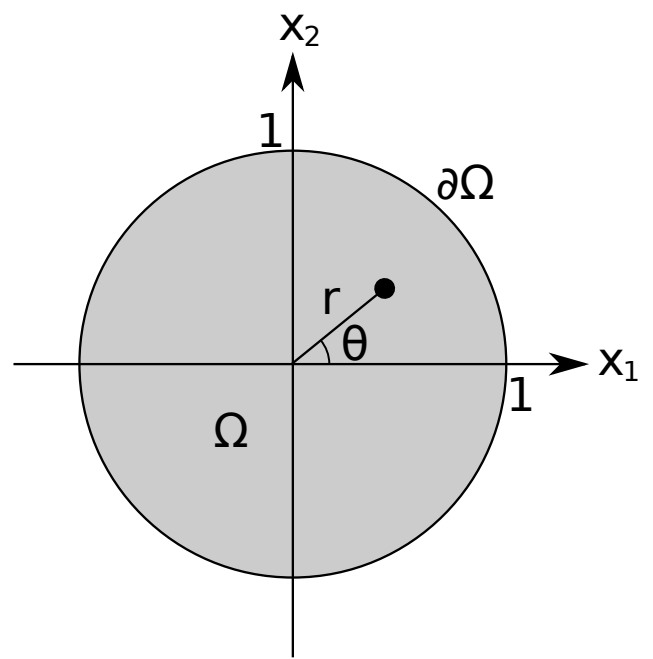

Figure 1. Coordinates $\left(x_{1}, x_{2}\right),(r, \theta)$ and domain $\Omega$ used to define the diffusion equation.

The set of equilibria of (1)-(2) is given by $\mathcal{E}=$ $\{\bar{\zeta}(r)=K \mid K \in \mathbb{R}\}$ (the origin plus a constant). Since we are only interested in the convergence of the solutions toward $\mathcal{E}$, we will consider hereafter the evolution of the variable $z \doteq \nabla \zeta \cdot \vec{\rho}$ (where $\vec{\rho}$ is the unit vector in the radial direction and $\nabla$ the gradient operator), as defined by:

$$
z_{t}=\left[\frac{\eta}{r}[r z]_{r}\right]_{r}+[\eta u]_{r}, \forall(r, t) \in(0,1) \times[0, T)
$$

with Dirichlet boundary conditions:

$$
\begin{aligned}
& z(0, t)=0, \forall t \in[0, T) \\
& z(1, t)=0, \forall t \in[0, T)
\end{aligned}
$$

and initial condition:

$$
z(r, 0)=z_{0}(r), \forall r \in(0,1)
$$

${ }^{2}$ Here $C^{\alpha_{c}, \beta_{c}}(\bar{\Omega} \times[0, T])$ denotes the space of functions which are $\alpha_{c}$-Hölder continuous in $\bar{\Omega}, \beta_{c}$-Hölder continuous in $[0, \mathrm{~T}] . P_{2}$ can be strengthened by assuming that $\eta$ and $u$ are in $C^{2,1}(\bar{\Omega} \times[0, T])$ which is the case for the physical application in Section $V$. where $z_{0} \doteq \nabla \zeta_{0} \cdot \vec{\rho}$.

The objectives of this paper are:

- To guarantee the exponential stability, in the topology of the $L^{2}$ norm $^{3}$, of solutions of equation (4) to zero, both in open-loop (with $u=0$ ) and by closing the loop with a controlled input $u(\cdot, t)$;

- to be able to adjust (in particular, to accelerate) the rate of convergence of the system using the controlled input;

- to determine the impact of a controller in the ISS gain in presence of a large class of errors. In particular actuation errors, estimation/measurement errors and state disturbances are considered.

To tackle this problem, a strict Lyapunov function will be defined in Section III. Let us state first an existence result assuming properties $P_{1}$ and $P_{2}$ :

Theorem 2.1: For every $z_{0}:[0,1] \rightarrow \mathbb{R}$ in $C^{2+\alpha_{c}}([0,1])$, $0<\alpha_{c}<1$, such that $z_{0}(0)=z_{0}(1)=0$, the evolution equations (4)-(6) have a unique solution $z \in C^{1+\alpha_{c}, 1+\alpha_{c} / 2}([0,1] \times$ $[0, T]) \cap C^{2+\alpha_{c}, 1+\alpha_{c} / 2}([0,1] \times[0, T])$.

The proof of this result is given in Appendix A.

\section{Control LyapunOV FunCtion AND Nominal STABILITY}

In this section, the input $u$ is considered to be perfectly controlled (without constraints) and a strict control Lyapunov function is developed, allowing us to construct a feedback law that ensures exponential convergence to the origin, at any desired rate, of the solutions of (4)-(6) in an $L^{2}$ sense.

\section{A. Candidate Control Lyapunov Function}

Given $f:[0,1] \rightarrow(0, \infty)$, a positive function with bounded second derivative, let us consider a candidate control Lyapunov function for the system (4) with boundary condition (5) and initial condition (6) defined, for all $z$ in $L^{2}([0,1])$, by:

$$
V(z(\cdot))=\frac{1}{2} \int_{0}^{1} f(r) z^{2}(r) d r
$$

Remark 3.1: Since $f(r)$ is positive and continuous on $[0,1]$, the weighted norm $\|z(\cdot)\|_{f} \doteq \sqrt{V(z(\cdot))}$ is equivalent to the usual $L^{2}$ norm. In particular, it verifies:

$$
\sqrt{\frac{f_{\min }}{2}}\|z(\cdot)\|_{L^{2}} \leq\|z(\cdot)\|_{f} \leq \sqrt{\frac{f_{\max }}{2}}\|z(\cdot)\|_{L^{2}}
$$

where $f_{\text {max }} \doteq \max _{r \in[0,1]} f(r)$ and $f_{\text {min }} \doteq \min _{r \in[0,1]} f(r)$.

Theorem 3.2: If there exist a positive function $f:[0,1] \rightarrow$ $(0, \infty)$ with bounded second derivative, and a positive constant $\alpha$ such that the following inequality is verified:

$$
\begin{array}{r}
\eta f^{\prime \prime}(r)+r\left[\frac{\eta}{r}\right]_{r} f^{\prime}(r)+\left[\frac{\eta}{r}\right]_{r} f(r) \leq-\alpha f(r), \\
\forall(r, t) \in[0,1] \times[0, T)
\end{array}
$$

then the time derivative $\dot{V}$ of the function $V$ defined by (7) verifies:

$$
\dot{V} \leq-\alpha V(z(\cdot, t))+\int_{0}^{1} f(r)[\eta u]_{r} z(r, t) d r, \forall t \in[0, T)
$$

${ }^{3}$ The $L^{p}$ norm of $\xi$ on a domain $\Omega$, will be denoted as $\|\xi\|_{L^{p}} \doteq$ $\left(\int_{\Omega} \xi^{p} d \Omega\right)^{1 / p}$ for $1<p<\infty$ 
along the solutions of (4), (5), (6).

Proof: Since Theorem 2.1 guarantees the existence of solutions to (4) such that $V(z(\cdot, t))$ is differentiable with respect to time, the derivative of $V$ along those trajectories is:

$$
\begin{aligned}
\dot{V} & =\int_{0}^{1} f(r) z z_{t} d r \\
& =T_{1}+T_{2}+T_{3}
\end{aligned}
$$

with:

$$
\begin{aligned}
T_{1} & =\int_{0}^{1} f(r)\left[\eta_{r} u+\eta u_{r}\right] z d r \\
T_{2} & =\int_{0}^{1} f(r)\left(\eta_{r}\left[z_{r}+\frac{1}{r} z\right] z+\eta\left[\frac{1}{r} z_{r}-\frac{1}{r^{2}} z\right] z\right) d r \\
T_{3} & =\int_{0}^{1} f(r) \eta z z_{r r} d r
\end{aligned}
$$

Term $T_{2}$ can be rewritten as:

$$
T_{2}=\int_{0}^{1} f(r)\left[\frac{1}{r} \eta z\right]_{r} z d r+\int_{0}^{1} f(r) \eta_{r} z z_{r} d r
$$

Integrating by parts we get:

$$
\begin{aligned}
T_{2}=\left.\frac{1}{r} f(r) \eta z^{2}\right|_{0} ^{1}-\int_{0}^{1} f^{\prime}(r) \eta \frac{1}{r} z^{2} d r & -\int_{0}^{1} f(r) \eta \frac{1}{r} z z_{r} d r \\
& +\int_{0}^{1} f(r) \eta_{r} z z_{r} d r
\end{aligned}
$$

and, using the boundary conditions (5), implies:

$$
\begin{aligned}
T_{2}=-\int_{0}^{1} f^{\prime}(r) \eta \frac{1}{r} z^{2} d r & -\int_{0}^{1} f(r) \eta \frac{1}{r} z z_{r} d r \\
& +\int_{0}^{1} f(r) \eta_{r} z z_{r} d r
\end{aligned}
$$

Integrating by parts $T_{3}$, the following equation is obtained:

$$
\begin{array}{r}
T_{3}=\left.f(r) \eta z z_{r}\right|_{0} ^{1}-\int_{0}^{1}\left(f^{\prime}(r) \eta\right. \\
\left.+f(r) \eta_{r}\right) z z_{r} d r \\
-\int_{0}^{1} f(r) \eta z_{r}^{2} d r
\end{array}
$$

which, considering again the boundary conditions (5), becomes:

$$
T_{3}=-\int_{0}^{1}\left(f^{\prime}(r) \eta+f(r) \eta_{r}\right) z z_{r} d r-\int_{0}^{1} f(r) \eta z_{r}^{2} d r
$$

From (12) and (13), (11) can thus be written as:

$$
\dot{V}=T_{1}+T_{4}-\int_{0}^{1} f^{\prime}(r) \eta \frac{1}{r} z^{2} d r-\int_{0}^{1} f(r) \eta z_{r}^{2} d r
$$

with:

$$
T_{4}=\int_{0}^{1}\left[-f(r) \eta \frac{1}{r}-f^{\prime}(r) \eta\right] z z_{r} d r
$$

Integrating by parts $T_{4}$, the following equation is obtained:

$$
\begin{aligned}
T_{4}= & \left.\frac{1}{2}\left(-f(r) \eta \frac{1}{r}-f^{\prime}(r) \eta\right) z^{2}\right|_{0} ^{1} \\
& -\frac{1}{2} \int_{0}^{1}\left(-f^{\prime}(r) \eta \frac{1}{r}-f(r) \eta_{r} \frac{1}{r}+f(r) \eta \frac{1}{r^{2}}\right. \\
& \left.-f^{\prime \prime}(r) \eta-f^{\prime}(r) \eta_{r}\right) z^{2} d r
\end{aligned}
$$

and the boundary conditions (5) imply that:

$$
\begin{array}{r}
T_{4}=\frac{1}{2} \int_{0}^{1}\left(f^{\prime}(r) \eta \frac{1}{r}+f(r) \eta_{r} \frac{1}{r}-f(r) \eta \frac{1}{r^{2}}+f^{\prime \prime}(r) \eta\right. \\
\left.+f^{\prime}(r) \eta_{r}\right) z^{2} d r
\end{array}
$$

Using (15), (14) is equivalent to:

$$
\begin{aligned}
\dot{V}= & \int_{0}^{1} f(r)\left[\eta_{r} u+\eta u_{r}\right] z d r-\int_{0}^{1} f(r) \eta z_{r}^{2} d r \\
& +\frac{1}{2} \int_{0}^{1}\left(-f^{\prime}(r) \eta \frac{1}{r}+f(r) \eta_{r} \frac{1}{r}-f(r) \eta \frac{1}{r^{2}}\right. \\
& \left.+f^{\prime \prime}(r) \eta+f^{\prime}(r) \eta_{r}\right) z^{2} d r
\end{aligned}
$$

From (9) and the definition of the Lyapunov candidate function, (16) provides the inequality:

$$
\begin{aligned}
& \dot{V} \leq-\alpha V(z(\cdot, t))+\int_{0}^{1} f(r)[\eta u]_{r} z d r-\int_{0}^{1} f(r) \eta z_{r}^{2} d r \\
& \forall t \in[0, T)
\end{aligned}
$$

which implies the inequality (10), thus concluding the proof of Theorem 3.2.

Remark 3.3: The last term in equation (17) can be bounded in order to obtain exponential stability of the system with a rate $\alpha+\epsilon$, where $\epsilon$ is a positive constant given by the application of Poincaré's inequality, the lower bound of $\eta$, and some bounds on $f$. However, for the physical application described in Section $V$, the rate of convergence obtained adding this term is almost the same as the value of $\alpha$ that can be obtained by adequately solving the differential inequality in Theorem 3.2.

Remark 3.4: For a large class of diffusivity profiles, the differential inequality in Theorem 3.2 has easily computable solutions: whenever $\eta_{r} \frac{1}{r}-\eta \frac{1}{r^{2}} \leq-k$ for some $k>0$ and all $(r, t) \in[0,1] \times[0, T)$ (for example, if the spatial derivative of the diffusivity coefficient remains non-positive), a constant $f$ satisfies (9). For our motivating application however, this condition is not satisfied. Section $V$ presents a suitable numerically computed weight satisfying (10) for the application. A heuristic method to compute such weights for the particular case of exponential diffusivity coefficient profiles is provided in [7].

\section{B. Some Implications}

Corollary 3.5: [Global Exponential Stability] If the conditions of Theorem 3.2 are verified, and if $u(r, t)=0$ for all $(r, t)$ in $[0,1] \times[0, T)$, then the origin of the system (4) with boundary conditions (5) and initial condition (6) is globally exponentially stable. The rate of convergence is $-\alpha / 2$ in the topology of the norm $L^{2}$, i.e.: $\|z(\cdot, t)\|_{L^{2}} \leq c e^{-\frac{\alpha}{2} t}\left\|z_{0}\right\|_{L^{2}}$ for a positive constant $c \doteq \sqrt{\frac{f_{\max }}{f_{\min }}}$, where $f_{\max }$ and $f_{\text {min }}$ are defined as in Remark 3.1, and for all $t \in[0, T)$.

Proof: From Theorem 3.2, and setting $u(r, t)=0$ for all $(r, t)$ in $[0,1] \times[0, T)$, the following inequality is obtained:

$$
\dot{V} \leq-\alpha V(z(\cdot, t)), \forall t \in[0, T)
$$


Therefore, considering the function $t \mapsto V(z(\cdot, t))$ and integrating the previous inequality over time implies that:

$$
V(z(\cdot, t)) \leq e^{-\alpha t} V\left(z_{0}(r)\right), \forall t \in[0, T)
$$

and consequently:

$$
\|z(\cdot, t)\|_{f} \leq e^{-\frac{\alpha}{2} t}\left\|z_{0}\right\|_{f}, \forall t \in[0, T)
$$

Since the norm $\|\cdot\|_{f}$ is equivalent to the usual $L^{2}$ norm ${ }^{4}$ as shown in Remark 3.1, Corollary 3.5 follows.

Corollary 3.6: [Convergence rate control] If the conditions of Theorem 3.2 are verified, and considering $u \doteq u_{c t r l}$ where $u_{\text {ctrl }}$ is chosen, for all $(r, t) \in(0,1) \times[0, T)$, as:

$$
u_{c t r l}(r, t)=-\frac{\gamma}{\eta} \int_{0}^{r} z(\rho, t) d \rho
$$

with $\gamma \geq 0$ a tuning parameter, then the system (4) with boundary conditions (5) and initial condition (6) is globally exponentially stable. Its convergence rate is $-\beta / 2 \doteq-(\alpha+$ $\gamma) / 2$, in the topology of the norm $L^{2}$.

The proof of this corollary is similar to that of Corollary 3.5, using Theorem 3.2 and the fact that $\left[\eta u_{c t r l}\right]_{r}=-\gamma z$ for all $(r, t) \in[0,1] \times[0, T)$.

\section{InPUT-TO-STATE StABILITY AND RobUstnesS}

Let us first consider the effect of disturbing equation (4) by including a term $w$ as follows:

$$
z_{t}=\left[\frac{\eta}{r}[r z]_{r}\right]_{r}+[\eta u]_{r}+w, \forall(r, t) \in(0,1) \times[0, T)
$$

where $w$ is a function of $(r, t)$ and the following property is assumed to hold:

$\mathbf{P}_{\mathbf{3}}$ : The two-dimensional Cartesian representation of $w$ belongs to $C^{\alpha_{c}, \alpha_{c} / 2}(\bar{\Omega} \times[0, T])$,

$0<\alpha_{c}<1$.

Proposition 4.1: [Disturbed version of Theorem 3.2] Let the conditions of Theorem 3.2 hold. Then, along the solution to (19), (5), (6), the following inequality holds:

$$
\begin{array}{r}
\dot{V} \leq-\alpha V(z(\cdot, t))+\int_{0}^{1} f(r)[\eta u]_{r} z d r+\int_{0}^{1} f(r) w z d r \\
\forall t \in[0, T)
\end{array}
$$

This fact follows from Theorem 3.2, by using (7) and noting that $\dot{V}_{\mid(19)}=\dot{V}_{\mid(4)}+\int_{0}^{1} f(r) w z d r$ where $\dot{V}_{\mid(19)}$ and $\dot{V}_{\mid(4)}$ stand for the derivative of $V$ along the solution of (19) and (4), respectively, with boundary conditions (5) and initial conditions (6).

Theorem 4.2: [ISS] Let the conditions of Proposition 4.1 be verified and consider $u \doteq u_{c t r l}$ as defined in Corollary 3.6. The following inequality holds for the evolution of the system (19) with boundary condition (5) and initial condition (6), for all $t \in[0, T)$ :

$$
\|z(\cdot, t)\|_{L^{2}} \leq c e^{-\frac{\beta}{2} t}\left\|z_{0}\right\|_{L^{2}}+c \int_{0}^{t} e^{-\frac{\beta}{2}(t-\tau)}\|w(\cdot, \tau)\|_{L^{2}} d \tau
$$

\footnotetext{
${ }^{4}$ For generality purposes, results in this article are stated in terms of usual norms. It should be noted, however, that the results stated in $\|\cdot\|_{f}$ norm are less conservative.
}

with $c=\sqrt{\frac{f_{\max }}{f_{\min }}}, f_{\max } \doteq \max _{r \in[0,1]} f(r)$ and $f_{\text {min }} \doteq$ $\min _{r \in[0,1]} f(r)$.

Proof: From Proposition 4.1 and Corollary 3.6 we have, along the solution of (19), (5), (6):

$$
\dot{V} \leq-\beta V(z(\cdot, t))+\int_{0}^{1}|f(r) w(r, t) z(r, t)| d r, \forall t \in[0, T)
$$

The function $f$ being positive and using the Cauchy-Schwarz inequality the following upper bound is obtained:

$$
\begin{aligned}
\dot{V} & \leq-\beta V(z(\cdot, t))+\|\sqrt{f} z(\cdot, t)\|_{L^{2}}\|\sqrt{f} w(\cdot, t)\|_{L^{2}} \\
& =-\beta V(z(\cdot, t))+2\|z(\cdot, t)\|_{f}\|w(\cdot, t)\|_{f}, \quad \forall t \in[0, T)
\end{aligned}
$$

Defining $X(z(\cdot, t)) \doteq \sqrt{V(z(\cdot, t))}=\|z(\cdot, t)\|_{f} \geq 0$ this inequality implies:

$$
\begin{array}{r}
2 X(z(\cdot, t)) \dot{X} \leq-\beta X^{2}(z(\cdot, t))+2 X(z(\cdot, t))\|w(\cdot, t)\|_{f}, \\
\forall t \in[0, T)
\end{array}
$$

where $\dot{X}=\frac{d}{d t} X(z(\cdot, t))$.

If $X(z)=0$, then $V(z)=0$ and $\dot{V}=0$. Otherwise we may divide both sides of the previous inequality by $2 X(z(\cdot, t))$ to get:

$$
\dot{X} \leq-\frac{\beta}{2} X(z(\cdot, t))+\|w(\cdot, t)\|_{f}, \forall t \in[0, T)
$$

From the last equation, by easy calculations, we get:

$$
\|z(\cdot, t)\|_{f} \leq e^{-\frac{\beta}{2} t}\left\|z_{0}\right\|_{f}+\int_{0}^{t} e^{-\frac{\beta}{2}(t-\tau)}\|w(\cdot, \tau)\|_{f} d \tau
$$

which in turn implies the desired result.

Corollary 4.3: [Actuation errors] In addition to the conditions in Theorem 3.2, we consider $u \doteq u_{c t r l}-\varepsilon^{u}(r, t)$, with $u_{\text {ctrl }}$ as defined in Corollary 3.6 and $\varepsilon^{u}(r, t)$ a distributed actuation error verifying the regularity conditions stated in $P_{2}$. Then, with $w \doteq 0$, the following inequality holds ${ }^{5}$ :

$$
\begin{aligned}
\|z(\cdot, t)\|_{L^{2}} \leq & c e^{-\frac{\beta}{2} t}\left\|z_{0}\right\|_{L^{2}} \\
& +c M \int_{0}^{t} e^{-\frac{\beta}{2}(t-\tau)}\left\|\varepsilon^{u}(\cdot, \tau)\right\|_{H^{1}} d \tau, \\
& \forall t \in[0, T)
\end{aligned}
$$

with $M \doteq \max \left\{\eta_{\max }, \eta_{r, \max }\right\}, \eta_{\max } \doteq \sup _{(r, t) \in[0,1] \times[0, T)} \mid$ $\eta \mid$ and $\eta_{r, \max } \doteq \sup _{(r, t) \in[0,1] \times[0, T)}\left|\eta_{r}\right|$.

The proof of Corollary 4.3 is directly obtained by replacing $w$ by $\left[\eta \varepsilon^{u}\right]_{r}$ in Theorem 4.2.

Corollary 4.4: [Estimation errors in the z profile] Assume that the conditions of Theorem 3.2 are verified and consider the control defined in Corollary 3.6 but substituting $z$ by an estimate, $\hat{z}(r, t) \doteq z(r, t)-\varepsilon^{z}(r, t)$ for all $(r, t) \in[0,1] \times[0, T)$, with $\varepsilon^{z}(r, t)$ being a distributed estimation error verifying the regularity conditions stated in $P_{3}$. The following inequality is then verified:

$$
\begin{aligned}
\|z(\cdot, t)\|_{L^{2}} \leq & c e^{-\frac{\beta}{2} t}\left\|z_{0}\right\|_{L^{2}} \\
& +\gamma c \int_{0}^{t} e^{-\frac{\beta}{2}(t-\tau)}\left\|\varepsilon^{z}(\cdot, \tau)\right\|_{L^{2}} d \tau \\
& \forall t \in[0, T)
\end{aligned}
$$

${ }^{5}$ The $H^{1}$ norm of $\xi$ on $[0,1]$, will be denoted as $\|\xi\|_{H^{1}} \doteq\|\xi\|_{L^{2}}+$ $\left\|\frac{\partial \xi}{\partial r}\right\|_{L^{2}}$ 
Corollary 4.4 follows readily by replacing $w$ by $\gamma \varepsilon^{z}$ in Theorem 4.2.

Proposition 4.5: [Estimation errors in the $\eta$ profile] Assume that the conditions of Theorem 3.2 are verified and consider the control defined in Corollary 3.6 but substituting $\eta$ by an estimate, $\hat{\eta}(r, t) \doteq \eta(r, t)-\varepsilon^{\eta}(r, t)$ for all $(r, t) \in$ $[0,1] \times[0, T)$, with $\varepsilon^{\eta}(r, t)$ being a distributed estimation error verifying the regularity conditions stated in $P_{2}$. The following inequality is then verified:

$$
\|z(\cdot, t)\|_{L^{2}} \leq c e^{-\frac{\beta^{\prime}}{2} t}\left\|z_{0}\right\|_{L^{2}}, \forall t \in[0, T)
$$

where $\beta^{\prime} \doteq \beta+\gamma \inf _{(r, t) \in[0,1] \times[0, T)}\left(\frac{\varepsilon^{\eta}}{\hat{\eta}}\right) \quad-$ $2 \gamma c \sup _{t \in[0, T)}\left\|\left[\frac{\varepsilon^{\eta}}{\hat{\eta}}\right]_{r}\right\|_{L^{2}}$.

Proof: Since the conditions of Theorem 3.2 are assumed to be verified to apply Corollary 3.6, inequality (10) holds. The control $u$ in Corollary 3.6 with $\hat{\eta}$ becomes:

$$
u=-\frac{\gamma}{\hat{\eta}} \int_{0}^{r} z(\rho, t) d \rho
$$

This implies:

$$
\begin{aligned}
\eta u & =-\gamma \frac{\eta}{\hat{\eta}} \int_{0}^{r} z(\rho, t) d \rho \\
& =-\gamma \frac{\hat{\eta}+\varepsilon^{\eta}}{\hat{\eta}} \int_{0}^{r} z(\rho, t) d \rho \\
& =-\gamma \int_{0}^{r} z(\rho, t) d \rho-\gamma \frac{\varepsilon^{\eta}}{\hat{\eta}} \int_{0}^{r} z(\rho, t) d \rho
\end{aligned}
$$

Differentiating with respect to the spatial variable:

$$
[\eta u]_{r}=-\gamma z-\gamma \frac{\varepsilon^{\eta}}{\hat{\eta}} z-\gamma\left[\frac{\varepsilon^{\eta}}{\hat{\eta}}\right]_{r} \int_{0}^{r} z(\rho, t) d \rho
$$

Substituting (26) in (10) the following inequalities are obtained for all $t \in[0, T)$ :

$$
\begin{aligned}
& \dot{V} \leq-\alpha V(z)-\gamma \int_{0}^{1} f(r) z^{2} d r-\gamma \int_{0}^{1} f(r) \frac{\varepsilon^{\eta}}{\hat{\eta}} z^{2} d r \\
& -\gamma \int_{0}^{1} f(r)\left[\frac{\varepsilon^{\eta}}{\hat{\eta}}\right]_{r}\left(\int_{0}^{r} z(\rho, t) d \rho\right) z d r \\
& \leq-(\alpha+\gamma) V(z) \\
& -\gamma\left[\inf _{(r, t) \in[0,1] \times[0, T)}\left(\frac{\varepsilon^{\eta}}{\hat{\eta}}\right)\right] \int_{0}^{1} f(r) z^{2} d r \\
& -\gamma \int_{0}^{1} f(r)\left[\frac{\varepsilon^{\eta}}{\hat{\eta}}\right]_{r}\left(\int_{0}^{r} z(\rho, t) d \rho\right) z d r \\
& \leq-\left(\beta+\gamma\left[\inf _{(r, t) \in[0,1] \times[0, T)}\left(\frac{\varepsilon^{\eta}}{\hat{\eta}}\right)\right]\right) V(z) \\
& +\gamma \int_{0}^{1}\left|f(r)\left[\frac{\varepsilon^{\eta}}{\hat{\eta}}\right]_{r}\left(\int_{0}^{r} z(\rho, t) d \rho\right) z\right| d r \\
& \leq-\left(\beta+\gamma\left[\inf _{(r, t) \in[0,1] \times[0, T)}\left(\frac{\varepsilon^{\eta}}{\hat{\eta}}\right)\right]\right) V(z) \\
& +\gamma \int_{0}^{1}\left|f(r)\left[\frac{\varepsilon^{\eta}}{\hat{\eta}}\right]_{r}\left(\int_{0}^{1}|z(\rho, t)| d \rho\right) z\right| d r \\
& \leq-\left(\beta+\gamma\left[\inf _{(r, t) \in[0,1] \times[0, T)}\left(\frac{\varepsilon^{\eta}}{\hat{\eta}}\right)\right]\right) V(z) \\
& +\gamma\|z\|_{L^{1}} \int_{0}^{1}\left|f(r)\left[\frac{\varepsilon^{\eta}}{\hat{\eta}}\right]_{r} z\right| d r
\end{aligned}
$$

Applying the Cauchy-Schwarz inequality on the integral term and on the $L^{1}$ norm of $z$ it implies that, for all $t \in[0, T)$ :

$$
\begin{aligned}
\dot{V} \leq-(\beta+\gamma & {\left.\left[\inf _{(r, t) \in[0,1] \times[0, T)}\left(\frac{\varepsilon^{\eta}}{\hat{\eta}}\right)\right]\right) V(z(\cdot, t)) } \\
& +2 \gamma\|z(\cdot, t)\|_{L^{2}}\left\|\left[\frac{\varepsilon^{\eta}}{\hat{\eta}}\right]_{r}\right\|_{f}\|z(\cdot, t)\|_{f}
\end{aligned}
$$

Using the equivalence between $\|\cdot\|_{f}$ and the usual $L^{2}$ norm, the previous inequality can be rewritten as:

$$
\begin{array}{r}
\dot{V} \leq-\left(\beta+\gamma\left[\inf _{(r, t) \in[0,1] \times[0, T)}\left(\frac{\varepsilon^{\eta}}{\hat{\eta}}\right)\right]\right) V(z(\cdot, t)) \\
+\frac{2 \sqrt{2} \gamma}{\sqrt{f_{\min }}}\|z(\cdot, t)\|_{f}^{2}\left\|\left[\frac{\varepsilon^{\eta}}{\hat{\eta}}\right]_{r}\right\|_{f}
\end{array}
$$

which in turn implies:

$$
\begin{array}{r}
\dot{V} \leq-\left(\beta+\gamma\left[\inf _{(r, t) \in[0,1] \times[0, T)}\left(\frac{\varepsilon^{\eta}}{\hat{\eta}}\right)\right]\right) V(z(\cdot, t)) \\
+2 \gamma c\|z(\cdot, t)\|_{f}^{2}\left\|\left[\frac{\varepsilon^{\eta}}{\hat{\eta}}\right]_{r}\right\|_{L^{2}}
\end{array}
$$

with $c$ as defined in Theorem 4.2. Consequently:

$$
\begin{aligned}
\dot{V} \leq & -\left(\beta+\gamma \inf _{(r, t) \in[0,1] \times[0, T)}\left(\frac{\varepsilon^{\eta}}{\hat{\eta}}\right)\right] \\
& \left.-2 \gamma c \sup _{t \in[0, T)}\left\|\left[\frac{\varepsilon^{\eta}}{\hat{\eta}}\right]_{r}\right\|_{L^{2}}\right) V(z(\cdot, t)), \forall t \in[0, T) \\
\leq & -\beta^{\prime} V(z(\cdot, t)), \forall t \in[0, T)
\end{aligned}
$$

and using the same arguments as in the proof of Corollary 3.5 it implies the desired result.

Remark 4.6: Although finding a stabilizing control law for system (4)-(6) considering unconstrained in-domain actuation is quite simple, the main interest of Sections III and IV lies in the fact that the stability of the open-loop system is guaranteed while giving a precise characterization of the impact of the control action in the closed-loop behaviour of the system, both in terms of rate of convergence and ISS gains. Furthermore, the fact that the ISS inequalities hold for the open-loop system is crucial for the application presented in Section $V$, since it also implies that stabilizing control laws can be found despite strong shape constraints on the admissible control action imposed by the physical actuators (represented in Section V-D by a nonlinear function of the two available engineering parameters in the LH antennas that can only take values in bounded sets).

\section{Application to the Control of the Poloidal Magnetic Flux Profile in a Tokamak Plasma}

\section{A. Physical Model}

Inside the toroidal chamber of a tokamak, the poloidal magnetic flux in the plasma, denoted $\psi(R, Z)$, is defined as the flux per radian of the magnetic field $\mathbf{B}(R, Z)$ through a disc centered on the toroidal axis at height $Z$, having a radius $R$ and surface $\mathbf{S}$, as depicted in Fig. 2. As the safety factor scales basically as the ratio of the normalized radius to poloidal magnetic gradient, controlling the latter allows controlling the safety 


\begin{tabular}{|c|c|c|}
\hline Variables & Description & Units \\
\hline$\psi$ & Poloidal magnetic flux profile & $T m^{2}$ \\
\hline$\phi$ & Toroidal magnetic flux profile & $T m^{2}$ \\
\hline$q$ & Safety factor profile $q \doteq d \phi / d \psi$ & \\
\hline$R_{0}$ & Location of the magnetic center & $m$ \\
\hline$B_{\phi_{0}}$ & Toroidal magnetic field at the center & $T$ \\
\hline$\rho$ & Equivalent radius of the magnetic surfaces & $m$ \\
\hline$a$ & Location of the last closed magnetic surface & $m$ \\
\hline$r$ & Normalized spatial variable $r \doteq \rho / a$ & \\
\hline$t$ & Time & $s$ \\
\hline$V$ & Plasma Volume & $m^{3}$ \\
\hline$F$ & Diamagnetic Function & $T m$ \\
\hline$C_{2}, C_{3}$ & Geometric coefficients & \\
\hline$\eta_{\|}$ & Parallel resistivity & $\Omega m$ \\
\hline$\eta$ & $\begin{array}{l}\text { Normalized diffusivity } \quad \text { coefficient } \\
\eta_{\|} /\left(\mu_{0} a^{2}\right)\end{array}$ & \\
\hline$\mu_{0}$ & Permeability of free space: $4 \pi \times 10^{-7}$ & $H m^{-1}$ \\
\hline$j_{n i}$ & Non-inductive effective current density & $A m^{-2}$ \\
\hline$j$ & $\begin{array}{l}\text { Normalized non-inductive effective current } \\
\text { density } \mu_{0} a^{2} R_{0} j_{n i}\end{array}$ & \\
\hline$j_{\phi}$ & Effective current density & $A m^{-2}$ \\
\hline$j_{\omega}$ & Inductive current density & $A m^{-2}$ \\
\hline$j_{\text {eccd }}$ & ECCD current density & $A m^{-2}$ \\
\hline$j_{l h}$ & LHCD current density & $A m^{-2}$ \\
\hline$j_{b s}$ & Bootstrap current density & $A m^{-2}$ \\
\hline$I_{p}$ & Total plasma current & A \\
\hline$P_{l h}$ & Lower Hybrid antenna power & $W$ \\
\hline$N_{\|}$ & Hybrid wave parallel refractive index & \\
\hline
\end{tabular}

Table I

VARIABLE DEFINITION

factor profile, which is an important physical heuristic that relates to the plasma Magnetohydrodynamic (MHD) stability and possible enhanced energy confinement. For a discussion on advanced tokamak scenarios, refer for instance to [31], [14], [38].

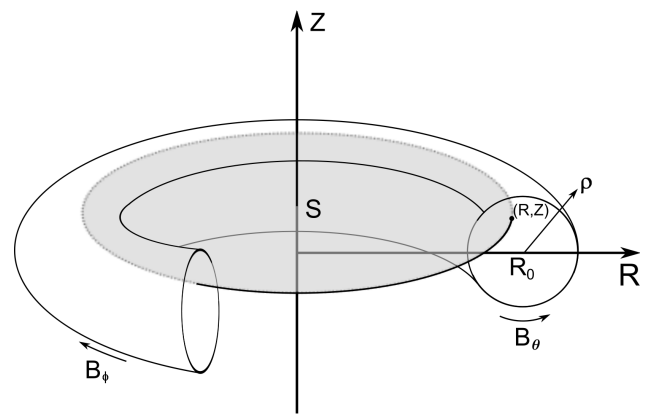

Figure 2. Coordinates $(R, Z)$ and surface $\mathbf{S}$ used to define the poloidal magnetic flux $\psi(R, Z)$.

In order to apply our analytical results, a simplified model for the magnetic flux profile $\psi$ in its one-dimensional representation is considered. Its dynamics are given by the following equation [4]:

$$
\psi_{t}=\frac{\eta_{\|} C_{2}}{\mu_{0} C_{3}} \psi_{\rho \rho}+\frac{\eta_{\|} \rho}{\mu_{0} C_{3}^{2}}\left(\frac{C_{2} C_{3}}{\rho}\right)_{\rho} \psi_{\rho}+\frac{\eta_{\|} V_{\rho} B_{\phi_{0}}}{F C_{3}} j_{n i}
$$

where $\rho \doteq \sqrt{\frac{\phi}{\pi B_{\phi_{0}}}}(\phi$ being the toroidal magnetic flux and $B_{\phi_{0}}$ the toroidal magnetic field at the center of the vacuum vessel) is an equivalent radius indexing the magnetic surfaces, $\eta_{\|}$is the parallel resistivity of the plasma, the source term $j_{n i}$ represents the current density profile generated by noninductive current sources, $\mu_{0}$ is the permeability of free space, $F$ is the diamagnetic function, $C_{2}$ and $C_{3}$ are geometric coefficients, $V_{\rho}$ is the spatial derivative of the plasma volume and $B_{\phi_{0}}$ is the toroidal magnetic field at the geometric center of the plasma. Some important variable definitions are given in Table I.

Neglecting the diamagnetic effect caused by poloidal currents and using a cylindrical approximation of the plasma geometry ( $\rho<<R_{0}$, where $R_{0}$ is the major plasma radius) the coefficients in (27) simplify as follows:

$$
F \approx R_{0} B_{\phi_{0}}, \quad C_{2}=C_{3}=4 \pi^{2} \frac{\rho}{R_{0}}, \quad V_{\rho}=4 \pi^{2} \rho R_{0}
$$

Defining a normalized spatial variable $r=\rho / a$, where $a$ (assumed constant) is the equivalent (minor) radius of the last closed magnetic surface, the simplified model is obtained as in [37], [2]:

$$
\psi_{t}(r, t)=\frac{\eta_{\|}(r, t)}{\mu_{0} a^{2}}\left(\psi_{r r}+\frac{1}{r} \psi_{r}\right)+\eta_{\|}(r, t) R_{0} j_{n i}(r, t)
$$

with the boundary conditions:

$$
\psi_{r}(0, t)=0
$$

and

$$
\psi_{r}(1, t)=-\frac{R_{0} \mu_{0} I_{p}(t)}{2 \pi}
$$

where $I_{p}$ is the total plasma current, and with the initial condition:

$$
\psi\left(r, t_{0}\right)=\psi_{0}(r)
$$

For the purposes of this article, $j_{n i}$ is considered as having one main component, which is the LHCD (Lower Hybrid Current Drive) current deposit $j_{l h}$. The extension to other non-inductive actuators is possible with minor modifications. Considering the evolution of the system around an equilibrium $(\bar{\psi}, \bar{j})$ and assuming an ideal tracking of the total plasma current, the evolution of $\psi$ is given by (1)-(3). Defining $z \doteq \nabla \tilde{\psi} \cdot \vec{\rho}, \eta \doteq \eta_{\|} /\left(\mu_{0} a^{2}\right)$ and $u \doteq \tilde{j}$, where $\tilde{\psi} \doteq \psi-\bar{\psi}$ and $\tilde{j} \doteq j-\bar{j}$, properties $P_{1}, P_{2}$ and $P_{3}$ hold, and thus the results of sections III and IV apply. Furthermore, the implementation of a state-feedback is possible due to the online availability of the magnetic flux profiles using the Equinox code, see [5].

\section{B. Illustration of Stability: Numerical computation of the Lyapunov function}

In order to test the proposed control law in Corollary 3.6 for the nominal system, we consider an identified estimate of the normalized plasma resistivity $\eta(r, t)=A(t) e^{\lambda(t) r}$, with $A(t) \doteq 0.0107-0.0014 \cos 40 \pi t$ and $\lambda(t) \doteq 6.1+0.8 \sin 20 \pi t$ for all $t \in[0, T)$. In particular $0.0093 \leq A(t) \leq 0.0121$ and $4.3 \leq \lambda(t) \leq 6.9$ for all $t \in[0, T)$. The limits for the variations were chosen from data extracted from Tore Supra shot 35109, described in [37]. A function $f$ satisfying the conditions of Theorem 3.2 for these values of $\eta$ has been numerically computed using Mathematica. It is depicted in Fig. 3. It should be noted that, in practice, the knowledge of these coefficients does not need to be exact. It is enough to find 


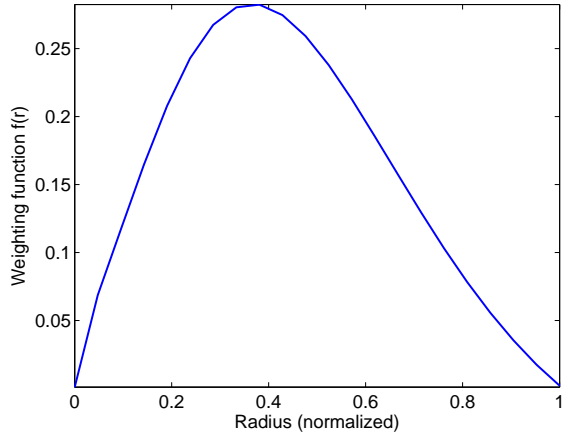

Figure 3. Function $f$ verifying the conditions of Theorem 3.2 for an exponential $\eta$ with time-varying parameters. $f_{\min }=0.001, f_{\max }=0.2823$.

a common weighting function $f$ valid on a rich enough set of profiles (and thus on convex combinations of those profiles). Moreover, since $\alpha$ in (9) is positive, it provides a robustness margin with respect to small numerical errors.

Using this $f$, the time-evolution of equation (4) with boundary conditions (5), initial condition (6) and of the associated Lyapunov function $V$ without control action $\left(u_{c t r l}=0\right)$, for an arbitrary numerical value of the initial condition, is shown in Fig. 4. The guaranteed convergence rate $\alpha$ is indeed respected but is conservative. This is not unexpected, since inequality (9) holds for all values of $r$ and the central and edge diffusivities vary considerably (almost by a factor 1000).

Finally, the response of the system using the control defined in Corollary 3.6, with $\gamma=1.6$ is shown in Fig. 5. Comparing Fig. 4 (c) and Fig. 5 (d) we can verify that the exponential decrease of $V$ with the control defined in Corollary 3.6 is indeed increased by at least $e^{-\gamma t}$, in agreement with the theoretical results.

\section{Illustration of ISS property: Tokamak Simulation with Unconstrained Controller}

In order to test the controller defined in Corollary 3.6 in a more realistic setting, not only considering the evolution of the diffusion equation but also the dynamics of the diffusivity coefficients and other system parameters, the simulator presented in [37] was used to test the behaviour of the system under the effect of disturbances and neglected inputs. In particular, the effect of the variation of the so-called bootstrap current (a plasma self-generated current source proportional to the inverse of the magnetic flux gradient that introduces a nonlinearity in the system dynamics) around the equilibrium and the Electron Cyclotron Current Drive (ECCD) input, turned on for $8 \mathrm{~s} \leq t \leq 20 \mathrm{~s}$, act as unknown exogeneous current sources in the evolution equation. For a rigorous treatment, they can be considered as disturbances both in the state and input (as in Theorem 4.2 and Corollary 4.3). The variation of the resistivity coefficients is caused mainly by variations in the temperature profile, which is affected by the LH antenna.

The original equilibrium was chosen from experimental data drawn from Tore Supra shot 35109. The effect of the ECCD antennas was overemphasized in order to better illustrate its action on the state and the Lyapunov function (the power in

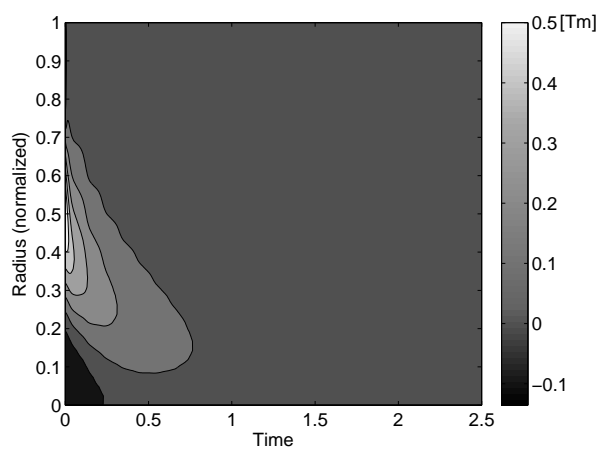

(a) Contour plot of the solution to the PDE.

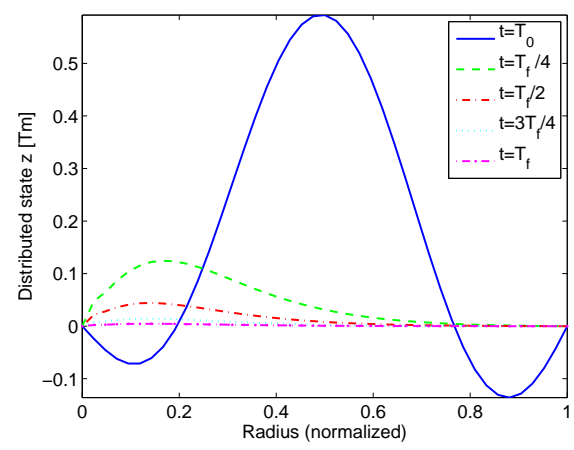

(b) Time-slices of the solution to the PDE.

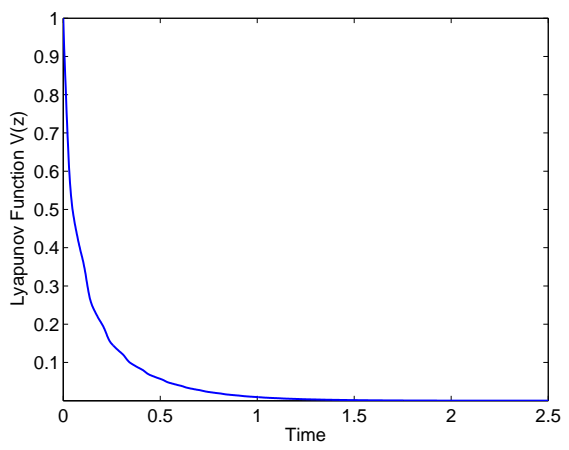

(c) Normalized evolution of the Lyapunov function.

Figure 4. Response of the nominal system without control action.

the simulation was chosen as three times the actual capacity of these actuators). A controller parameter $\gamma=0.75$ was found to yield acceptable results (both in terms of the amplitude of the control and the effect of the noisy measurements in the system). The results are shown in Fig. 6, with control action starting at $t=16 \mathrm{~s}$. While a steady-state error remains when the ECCD is turned on, it is significantly reduced by the feedback action. The convergence speed is also noticeably improved.

\section{Exploiting the Lyapunov Approach: Tokamak Simulation with Constrained Controller}

In view of a possible implementation of the control law in a real tokamak experiment, strict constraints have to be imposed on the control action. For this application, the actuator considered is the current current density generated by the 


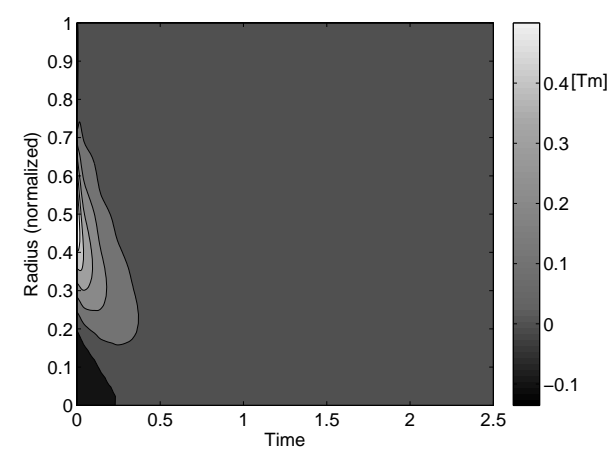

(a) Contour plot of the solution to the PDE.

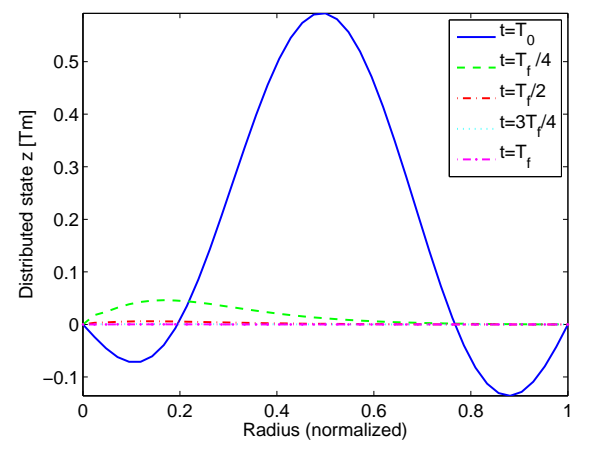

(b) Time-slices of the solution to the PDE.

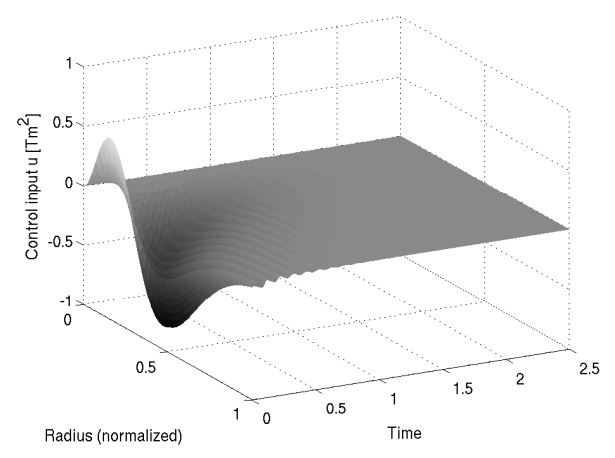

(c) Evolution of the control $u$.

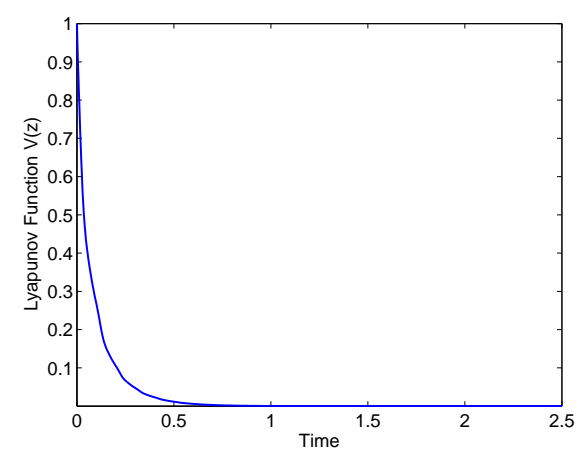

(d) Normalized evolution of the Lyapunov function.

Figure 5. Response of the nominal system with unconstrained control action $(\gamma=1.6)$

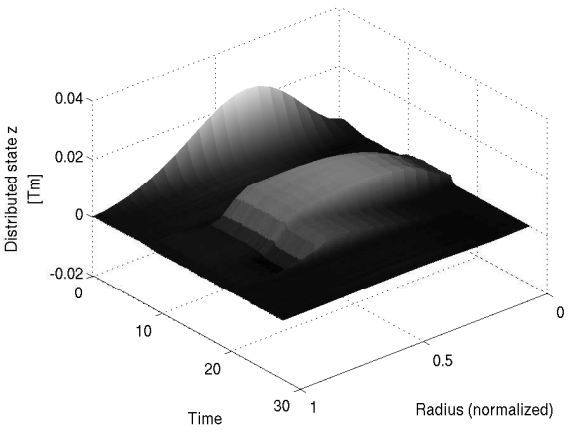

(a) Evolution of the $\mathrm{z}$ profile in time.

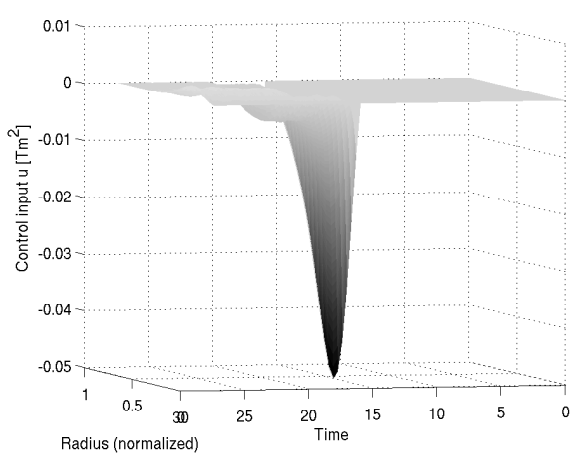

(b) Evolution of the control $u$.

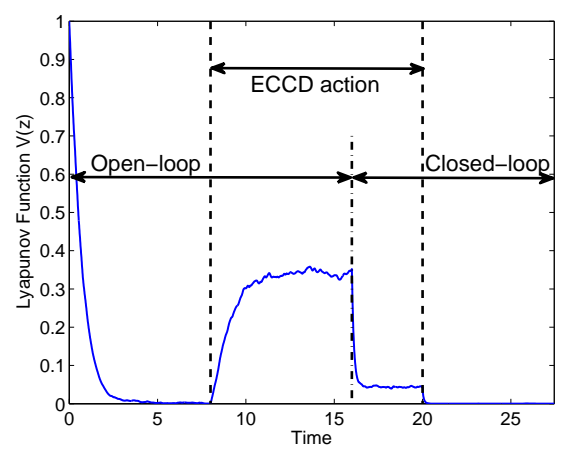

(c) Normalized evolution of the Lyapunov function.

Figure 6. Response of the disturbed system, disturbance applied at $t=8$ $\mathrm{s}$ and removed at $t=20 \mathrm{~s}$ with unconstrained control action beginning at $t=16 \mathrm{~s}(\gamma=0.75)$.

lower hybrid waves. This current deposit profile $j_{l h}(r, t)$ depends on two main physical parameters: the power delivered by the antennas $P_{l h}(t)$ and the parallel refractive index $N_{\|}(t)$. In Tore Supra two LH antennas exist and their parameters may vary in the following manner: $P_{l h, 1} \leq 1.5 \mathrm{MW}, P_{l h, 2} \leq 3$ MW, $N_{\|, 1} \in[1.43,2.37]$ and $N_{\|, 2} \in[1.67,2.33]$. However, in this paper only one set of parameters $\left(P_{l h}, N_{\|}\right)$is used to derive a controller that illustrates the usefulness of the control Lyapunov function, as defined in Proposition 4.1, from a practical standpoint.

Based on Proposition 4.1, we propose to choose, at each 
time step, a couple $\left(P_{l h}^{*}, N_{\|}^{*}\right)$ as follows:

$$
\left(P_{l h}^{*}, N_{\|}^{*}\right)=\arg \min _{\left(P_{l h}, N_{\|}\right) \in \mathcal{U}} \int_{0}^{1} f(r)\left[\eta u\left(P_{l h}, N_{\|}\right)\right]_{r} z d r
$$

subject to the constraints:

$$
0 \geq \int_{0}^{1} f(r)\left[\eta u\left(P_{l h}^{*}, N_{\|}^{*}\right)\right]_{r} z d r \geq-\gamma V(z)
$$

where $\mathcal{U} \doteq\left[P_{l h, \text { min }}, P_{l h, \max }\right] \times\left[N_{\|, \min }, N_{\|, \max }\right]$ and $u$ : $\mathcal{U} \rightarrow C^{\infty}([0,1])$ is a nonlinear function representing the relation between the engineering parameters and the variations in the $j_{l h}$ profile as presented in [37].

Remark 5.1: The inequality in the left-hand side of (32) guarantees that the worst case of the optimization scheme is $\int_{0}^{1} f(r)\left[\eta u\left(P_{l h}^{*}, N_{\|}^{*}\right)\right]_{r} z d r=0$. In other words, the closedloop system verifies the ISS inequalities of Theorem 4.2 and Corollary 4.3 for a value of $\beta \geq \alpha$. The inequality in the righthand side of (32) is not necessary for the stability of system (4)-(6), but aims to limit the contribution of the controller on the rate of convergence of the closed-loop system. If, for all time, there exist $\left(P_{l h}, N_{\|}\right) \in \mathcal{U}$ such that the control proposed in Corollary 3.6 is exactly $u\left(P_{l h}, N_{\|}\right)$, then it is a solution to the constrained optimization problem.

Since solving this optimization problem analytically is quite difficult, a numerical method using a gradient-descent algorithm on the discretized parameter space was implemented in practice. As the state dynamics describe the system deviation from an equilibrium, choosing $u=0$ (i.e. $\left(P_{l h}, N_{\|}\right)=$ $\left(\bar{P}_{l h}, \bar{N}_{\|}\right)$) always gives a feasible starting point. In general, we might not find a solution of the proposed problem (31), and we could have problems facing local-minima, but under simulation with data taken from Tore Supra shots 35109 and 31463 (the first generated by modulating the LH power, the second including also ECCD action) the results are satisfying.

The values of $u$ and $u_{r}$ for the different vertices of the parameter grid were calculated off-line to allow real-time control. In this case, the mean time taken by the algorithm to determine the control values was $432 \mu$ s using a Matlab ${ }^{\circledR}$ function running on a processor at $2.54 \mathrm{GHz}$.

For the first simulation, using an equilibrium point taken from Tore Supra shot 35109, we introduce a disturbance as in the previous section, corresponding to three times the maximum ECCD power for $8 \mathrm{~s} \leq t \leq 20 \mathrm{~s}$ and then activate the control at $t=16 \mathrm{~s}$ to attenuate its effect. Results are shown in Fig. 7. It can be seen that, despite the constraints, the attenuation of the disturbance is very effective, with the value of the Lyapunov function rapidly reduced once the feedback control is activated. The control value was updated every 0.1 $\mathrm{s}$, which is much greater than the required computing time.

The second proposed scenario is a change of operating point, where both equilibria were drawn from Tore Supra shot 35109. Control action starts at $t=4 \mathrm{~s}$ and the change of reference is applied at $t=17 \mathrm{~s}$. The results can be seen in Fig. 8. It is interesting to see the behaviour of the Lyapunov function under the constrained control: even though an exponentially decreasing upper bound exists, the actual shape is more irregular than in the unconstrained case (similar

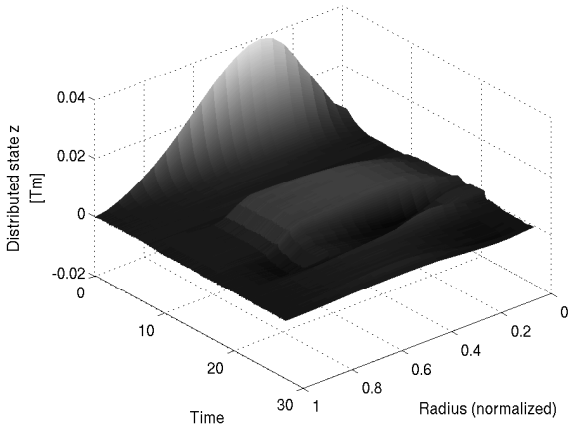

(a) Evolution of the $\mathrm{z}$ profile in time.

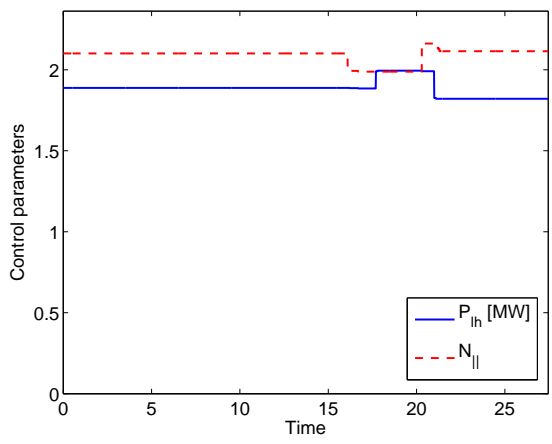

(b) Antenna parameters used to calculate the control input.

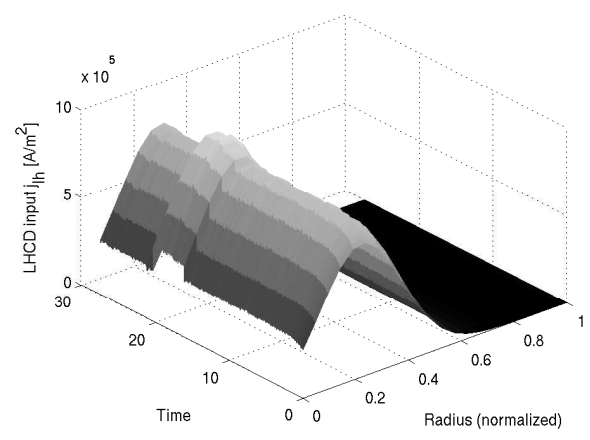

(c) Evolution of the actual $j_{l h}$ applied to the system.

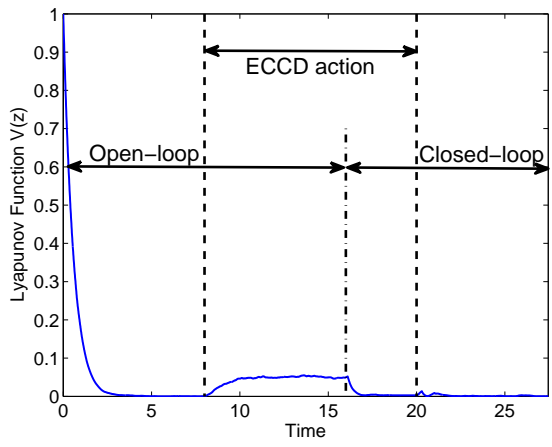

(d) Normalized evolution of the Lyapunov function.

Figure 7. Response of the disturbed system, disturbance applied at $t=8 \mathrm{~s}$ and removed at $t=20 \mathrm{~s}$ with constrained control action beginning at $t=16$ $\mathrm{s}(\gamma=0.6)$ 
to a time-varying gain guaranteeing at all times a negative derivative for the Lyapunov function).

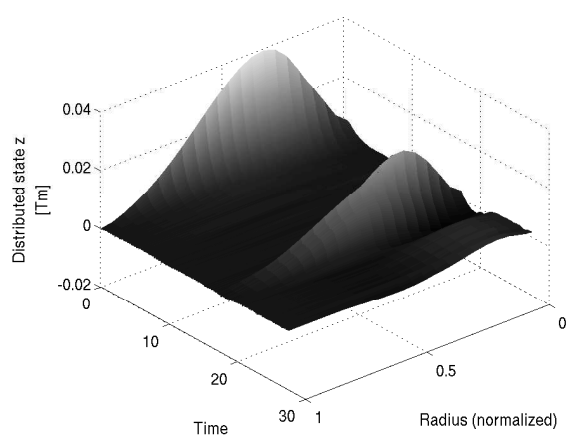

(a) Evolution of the $\mathrm{z}$ profile in time.

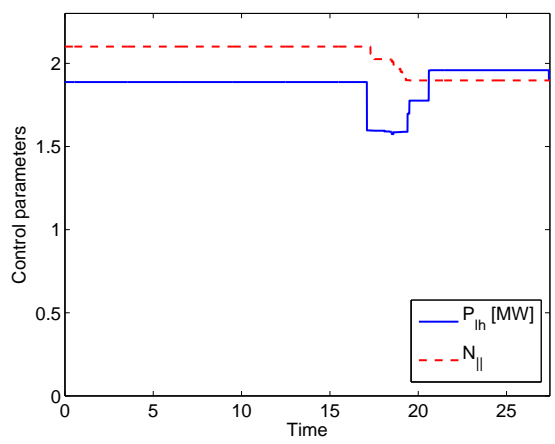

(b) Antenna parameters used to calculate the control input.

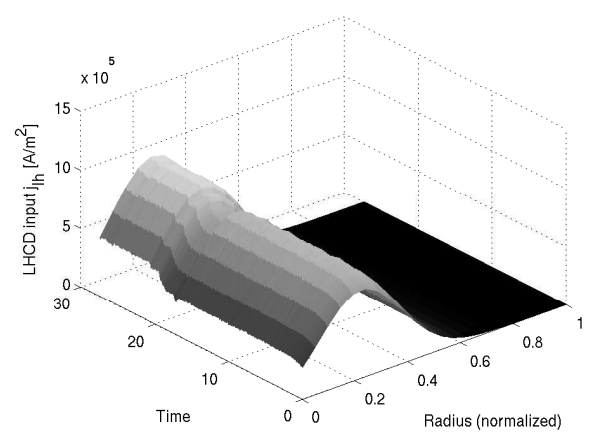

(c) Evolution of the actual $j_{l h}$ applied to the system.

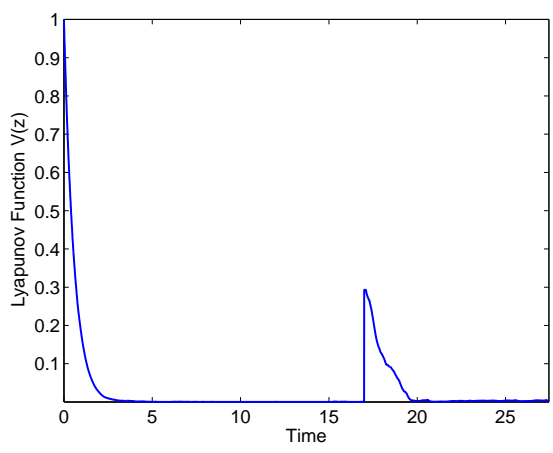

(d) Normalized evolution of the Lyapunov function.

Figure 8. Response of the system, change of reference applied at $t=17 \mathrm{~s}$ with constrained control action beginning at $t=4 \mathrm{~s}(\gamma=0.6)$.
Finally, a more complicated tracking scenario is proposed, where a time-varying reference is generated from Tore Supra shot 31463 (which involves both LH and ECCD action). Furthermore, only one equilibrium point is calculated, corresponding to the mean value of the parameters applied during the shot instead of one for each point of the trajectory. Fig. 9 represents (a) the mean tracking error, (b) the values for the engineering parameters of the LH antenna, (c) the LH current deposit profile, and (d) the safety factor profile. This result illustrates the robustness of the controller with respect to deviations from the calculated equilibrium (used in the computation of the feedback).

\section{CONCLUSION}

In this paper, a strict Lyapunov function was found for a diffusion equation with time-varying distributed coefficients. This function guarantees some ISS properties for the system and allows for the construction of simple control laws that maintain these properties and improve the performance of the system. A particularly important contribution was a robustness study of the system with respect to disturbances and errors in the model and measurements, since for most physical applications the exact values and behaviour of the diffusivity coefficients is not well known. Another contribution is the consideration of the distributed and time-varying nature of these coefficients in the nominal scenario without constraining their rate of variation. Finally, the proposed Lyapunov function design was applied to the control of the gradient of the poloidal magnetic flux profile in the Tore Supra tokamak, with the objective of safety factor regulation.

Future work will be devoted to the implementation and testing of the proposed constrained control law with a more complex simulation code, METIS and/or CRONOS, see [1], [2] respectively. These codes include energy and momentum conservation laws as well as refined plasma/wave interaction descriptions for the antennas. Some effort will also be devoted to the estimation of the diffusivity coefficients in view of an experimental implementation on Tore Supra.

\section{APPENDIX}

Proof of Theorem 2.1: This proof is organized as follows:

(a) First, an auxiliary problem in two-dimensional Cartesian coordinates under symmetry conditions is formulated.

(b) Next, the existence and uniqueness of solutions to the auxiliary problem is shown using Theorem 5.1.21 and Corollary 5.1.22 in [18] (pages 206-208). Which in turn imply the existence and uniqueness of solutions to the problem (4)-(6).

(a): Consider the following two-dimensional Cartesian auxiliary system:

$$
\begin{array}{r}
\zeta_{t}\left(x_{1}, x_{2}, t\right)=\eta\left(x_{1}, x_{2}, t\right) \Delta \zeta\left(x_{1}, x_{2}, t\right)+F\left(x_{1}, x_{2}, t\right), \\
\forall\left(x_{1}, x_{2}, t\right) \in \Omega \times[0, T)
\end{array}
$$

with symmetric boundary condition:

$$
\zeta_{\nu}\left(x_{1}, x_{2}, t\right)=0, \quad \forall\left(x_{1}, x_{2}, t\right) \in \partial \Omega \times[0, T)
$$




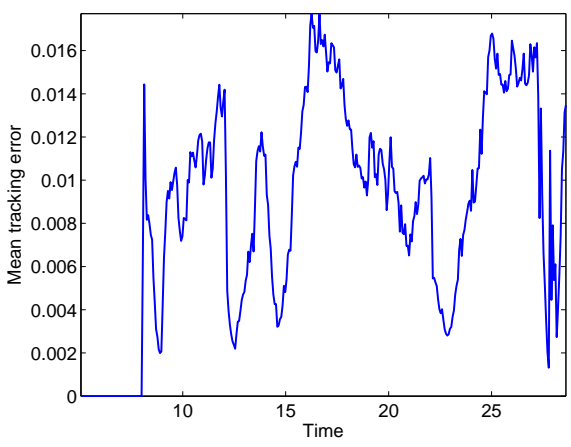

(a) Evolution of the normalized mean error in time.

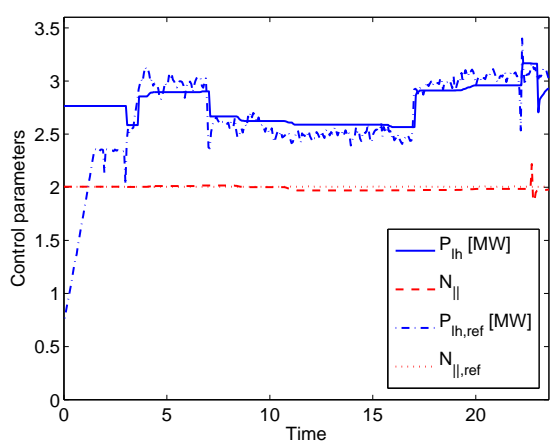

(b) Antenna parameters used to calculate the control input.

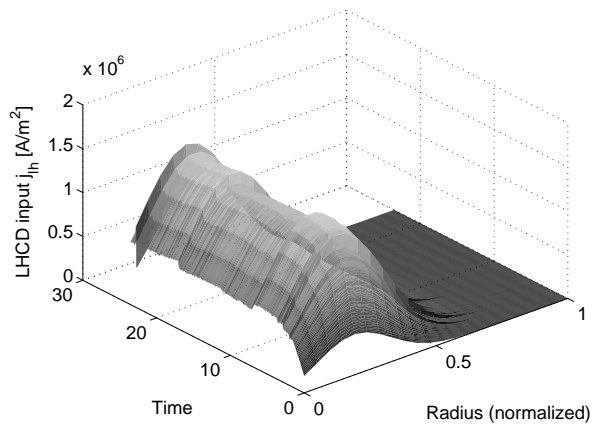

(c) Evolution of the actual $j_{l h}$ applied to the system.

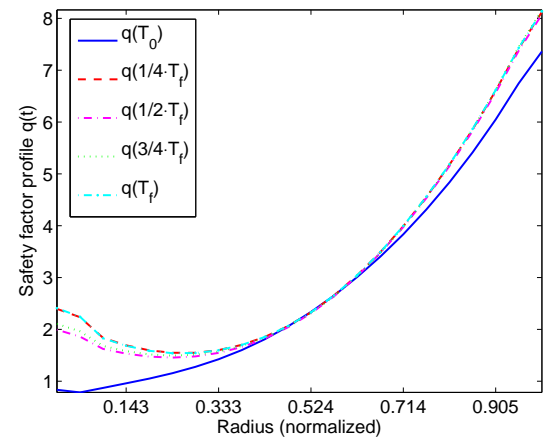

(d) Evolution of the safety factor profile.

Figure 9. Response of the system, with constrained control action beginning at $t=3.1 \mathrm{~s}(\gamma=2.5)$. where $\zeta_{\nu}$ is the derivative of $\zeta$ in the outward normal direction to $\partial \Omega$, and with symmetric initial condition $\zeta_{0} \in \mathcal{C}^{3+\alpha_{c}}(\bar{\Omega})$, $0<\alpha_{c}<1$ :

$$
\zeta\left(x_{1}, x_{2}, 0\right)=\zeta_{0}\left(x_{1}, x_{2}\right), \quad \forall\left(x_{1}, x_{2}\right) \in \Omega
$$

where $\Delta$ is the Laplacian, $F\left(x_{1}, x_{2}, t\right) \doteq$ $\eta\left(x_{1}, x_{2}, t\right) u\left(x_{1}, x_{2}, t\right)$. This system is equivalent, when imposing a central symmetry condition and sufficient regularity of the initial condition, to (1)-(3).

(b): To apply Theorem 5.1.21 and Corollary 5.1.22 in [18] (pages 206-208) it must be shown first that the diffusive operators verify a uniform ellipticity condition in $\bar{\Omega}$. This is trivially verified as a direct consequence of $P_{1}$ and therefore Theorem 5.1.21 gives the existence and uniqueness of solutions and Corollary 5.1.22 establishes the desired regularity (such that the gradient is in $C^{2+\alpha_{c}, 1+\alpha_{c} / 2}(\bar{\Omega} \times[0, T])$ ). This degree of regularity is sufficient to ensure that all the integrals used for the definition of the Lyapunov function and its time derivative are well defined. This concludes the proof of Theorem 2.1.

Existence, uniqueness and regularity results are also valid when the control input is of the form proposed in Corollary 3.6 (which amounts to a feedback in the variable $\zeta$ ). and can extend to certain forms of non-homogeneous boundary conditions thanks to the structure of the operators considered in [18].

\section{REFERENCES}

[1] J. F. Artaud. METIS user's guide. CEA/IRFM/PHY/NTT-2008.001, 2008.

[2] J. F. Artaud et al. The CRONOS suite of codes for integrated tokamak modelling. Nucl. Fusion, 50, 043001, 2010.

[3] R. A. Baker and A. R. Bergen. Lyapunov stability and Lyapunov functions of infinite dimensional systems. IEEE Transactions on Automatic Control, 14(4):325-334, August 1969.

[4] J. Blum. Numerical Simulation and Optimal Control in Plasma Physics. Wiley/Gauthier-Villars Series in Modern Applied Mathematics. Gauthier-Villars, John Wiley \& Sons, 1989.

[5] J. Blum, C. Boulbe, and B. Faugeras. Reconstruction of the equilibrium of the plasma in a tokamak and identification of the current density profile in real time. Journal of Computational Physics, In Press, 2011.

[6] F. Bribiesca Argomedo, C. Prieur, E. Witrant, and S. Brémond. Polytopic control of the magnetic flux profile in a tokamak plasma. In Proceedings of the 18th IFAC World Congress. Milan, Italy, 2011.

[7] F. Bribiesca Argomedo, E. Witrant, and C. Prieur. Input-to-state stability of a time-varying nonhomogeneous diffusive equation subject to boundary disturbances. In Proceedings of the American Control Conference, Montréal, Canada, 2012.

[8] T. Cazenave and A. Haraux. An introduction to semilinear evolution equations. Oxford University Press, 1998.

[9] J.-M. Coron, G. Bastin, and B. d'Andréa Novel. Dissipative boundary conditions for one-dimensional nonlinear hyperbolic systems. SIAM Journal on Control and Optimization, 47(3):1460-1498, 2008.

[10] J.-M. Coron and B. d'Andréa Novel. Stabilization of a rotating body beam without damping. IEEE Transactions on Automatic Control, 43(5):608-618, May 1998.

[11] J.-M. Coron, B. d'Andréa Novel, and G. Bastin. A strict Lyapunov function for boundary control of hyperbolic systems of conservation laws. IEEE Transactions on Automatic Control, 52(1):2-11, January 2007.

[12] A. Gahlawat, M. M. Peet, and E. Witrant. Control and verification of the safety-factor profile in tokamaks using sum-of-squares polynomials. In Proceedings of the 18th IFAC World Congress. Milan, Italy, August, 2011.

[13] O. Gaye, E. Moulay, S. Brémond, L. Autrique, R. Nouailletas, and Y. Orlov. Sliding mode stabilization of the current profile in tokamak plasmas. In Proceedings of the 50th IEEE Conference on Decision and Control and European Control Conference, pages 2638-2643, 2011. 
[14] C. Gormezano. High performance tokamak operation regimes. Plasma Phys. Control. Fusion, 41:B367-80, 1999.

[15] B. Jayawardhana, H. Logemann, and E. P. Ryan. Infinite-dimensional feedback systems: the circle criterion and input-to-state stability. Communications in Information and Systems, 8(4):413-444, 2008.

[16] M. Krstic and A.T. Smyshlyaev. Adaptive boundary control for unstable parabolic PDEs-part I: Lyapunov design. IEEE Transactions on Automatic Control, 53(7):1575-1591, August 2008.

[17] L. Laborde et al. A model-based technique for integrated real-time profile control in the JET tokamak. Plasma Phys. Control. Fusion, 47:155-183, 2005.

[18] A. Lunardi. Analytic Semigroups and Optimal Regularity in Parabolic Problems, volume 16 of Progress in nonlinear differential equations and their applications. Birkhäuser, 1995.

[19] F. Mazenc and C. Prieur. Strict Lyapunov functions for semilinear parabolic partial differential equations. Mathematical Control and Related Fields, 1:231-250, 2011.

[20] D. Moreau et al. Real-time control of the q-profile in JET for steady state advanced tokamak operation. Nucl. Fusion, 43:870-882, 2003.

[21] P. Moreau et al. Plasma control in Tore Supra. Fusion Science and Technology, 56:1284-1299, October 2009.

[22] Y. Ou, C. Xu, E. Schuster, T. C. Luce, J. R. Ferron, M. L. Walker, and D. A. Humphreys. Optimal tracking control of current profile in tokamaks. IEEE Transactions on Control Systems Technology, 19(2):432-441, March 2011.

[23] M. M. Peet, A. Papachristodoulou, and S. Lall. Positive forms and stability of linear time-delay systems. SIAM Journal on Control and Optimization, 47(6):3237-3258, 2009.

[24] A. Pironti and M. Walker. Control of tokamak plasmas. IEEE Control System Magazine, 25(5) 2005.

[25] C. Prieur and F. Mazenc. ISS-Lyapunov functions for time-varying hyperbolic systems of balance laws. Math. Control Signals Systems, 24(1):111-134, 2012.

[26] A. Smyshlyaev and M. Krstic. Closed-form boundary state feedback for a class of 1-D partial integro-differential equations. IEEE Transactions on Automatic Control, 49(12):2185-2201, 2004.

[27] A. Smyshlyaev and M. Krstic. On control design for PDEs with space-dependent diffusivity or time-dependent reactivity. Automatica, 41:1601-1608, 2005.

[28] A. Smyshlyaev and M. Krstic. Adaptive boundary control for unstable parabolic PDEs-part II: Estimation-based designs. Automatica, 43(9):1543-1556, 2007.

[29] A. Smyshlyaev and M. Krstic. Adaptive boundary control for unstable parabolic PDEs-part III: Output feedback examples with swapping identifiers. Automatica, 43(9):1557-1564, 2007.

[30] E. D. Sontag. Input to state stability: Basic concepts and results. In Nonlinear and Optimal Control Theory, pages 163-220. SpringerVerlag, Berlin, 2007.

[31] T. S. Taylor. Physics of advanced tokamaks. Plasma Phys. Control. Fusion, 39:B47-73, 1997.

[32] R. Vazquez and M. Krstic. Explicit integral operator feedback for local stabilization of nonlinear thermal convection loop PDEs. Systems \& Control Letters, 55:624-632, 2006.

[33] R. Vazquez and M. Krstic. Control of Turbulent and Magnetohydrodynamic Channel Flows Boundary Stabilization and State Estimation. Systems \& Control: Foundations \& Applications. Birkhäuser, 2008.

[34] R. Vazquez and M. Krstic. Boundary observer for output-feedback stabilization of thermal-fluid convection loop. IEEE Transactions on Control Systems Technology, 18(4):789-797, 2010.

[35] M.L. Walker, D.A. Humphreys, D. Mazon, D. Moreau, M. Okabayashi, T.H. Osborne, and E. Schuster. Emerging applications in tokamak plasma control. IEEE Control Systems Magazine, 26(2):35-63, 2006.

[36] J. Wesson. Tokamaks. International Series of Monographs on Physics 118. Oxford University Press, third edition, 2004

[37] E. Witrant, E. Joffrin, S. Brémond, G. Giruzzi, D. Mazon, O. Barana, and P. Moreau. A control-oriented model of the current control profile in tokamak plasma. Plasma Phys. Control. Fusion, 49:1075-1105, 2007.

[38] R. C. Wolf. Internal transport barriers in tokamak plasmas. Plasma Phys. Control. Fusion, 45:R1-91, 2003.

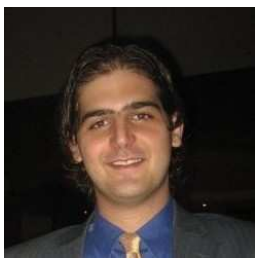

Federico Bribiesca Argomedo was born in Zamora, Michoacán, Mexico in 1987. He obtained a B.Sc. in Mechatronics Engineering from the Tecnológico de Monterrey, Monterrey, Mexico in 2009 and a M.Sc. from Grenoble INP in 2009. He is currently a Ph.D. candidate in Control Systems working at GIPSA-lab (Grenoble University). His research interests include nonlinear control theory and control of partial differential equations. In particular, he has applied these techniques to tokamak safety factor control.

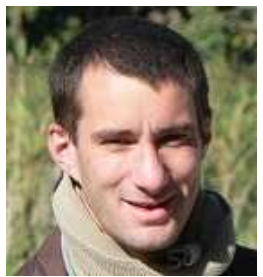

Christophe Prieur was born in Essey-les-Nancy, France, in 1974. He graduated in Mathematics from the Ecole Normale Supérieure de Cachan, France in 2000. He received the Ph.D degree in 2001 in Applied Mathematics from the Université ParisSud, France, and the "Habilitation à Diriger des Recherches" (HDR degree) in 2009. From 2002 he was an associate researcher CNRS at the laboratory SATIE, Cachan, France, and at the LAAS, Toulouse, France (2004-2010). In 2010 he joined the Gipsalab, Grenoble, France where he is currently a senior researcher of the CNRS (since 2011). His current research interests include nonlinear control theory, hybrid systems, and control of nonlinear partial differential equations.

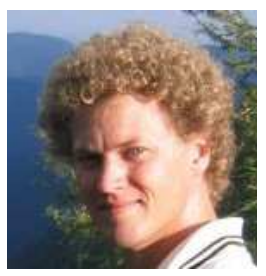

Emmanuel Witrant obtained a B.Sc. in Aerospace Engineering from Georgia Institute of Technology in 2001 and a Ph.D. in Automatic Control from Grenoble University in 2005. He joined University Joseph Fourier and GIPSA-lab as an Associate Professor in 2007. His research interest is focused on the modeling and control of inhomogeneous transport phenomena (information, energy, gases...), with real-time and optimization constraints. The resulting methods provide new results for controlled thermonuclear fusion, environmental sciences and

Poiseuille's flows.

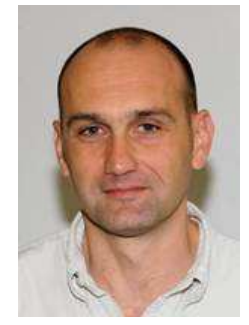

Sylvain Brémond obtained a Master degree in Electrical Engineering from the Ecole Supérieure d'Electricité (Supélec), France, in 1991 and a Ph.D. in Automatic control from the Université Paris-Sud, France, in 1995. From 1995 he was a member of the Institut de Recherches sur la Fusion par confinement Magnétique (IRFM), the CEA laboratory in charge of magnetic fusion research, where he has been successively leading the Tore Supra tokamak main heating facility operation team and the plasma control and machine operation group. His current research interests include tokamak operation and plasma control. 